\title{
Social Entrepreneurship im deutschen Wohlfahrtsstaat - Hybride Organisationen zwischen Markt, Staat und Gemeinschaft
}

\author{
Rolf G. Heinze, Katrin Schneiders und Stephan Grohs
}

\section{$1 \quad$ Einleitung}

Der deutsche Wohlfahrtsstaat galt lange Zeit als Idealtypus des konservativen Wohlfahrtsstaates mit einem dominierenden Sozialversicherungsprinzip, einer Dezentralisierung residualer Leistungen und stark korporatistisch geprägten Dienstleistungsstrukturen (Schmid 2010). Der „Markt“ nahm in diesen Arrangements nur eine marginale Bedeutung ein. Einige der zentralen Organisationsprinzipien gerieten im vergangenen Jahrzehnt jedoch durch Finanzierungsprobleme und den Einfluss neuer Deutungsschemata unter Veränderungsdruck (Heinze 2009).

Vor diesem Hintergrund gewinnen auch Diskussionen um neue „Spieler“ in der Produktion sozialer Dienstleistungen an Vehemenz. Neben der in diesem Buch betrachteten Bedeutung von social entrepreneurship [ebenso wie Sozialunternehmen und Sozialunternehmer im Sinne von social entrepreneurship organization im Folgenden überwiegend mit dem Kürzel SEO bezeichnet] spielen Diskussionen um Sozialunternehmertum, Privatisierung und Public Private Partnerships eine zunehmende Rolle. Wie diese neuen Akteure in etablierte Strukturen der Wohlfahrtsproduktion eingebunden werden, wird in der sozialwissenschaftlichen Forschung dagegen noch unzureichend behandelt. Vor diesem Hintergrund werden in diesem Beitrag folgende Fragen aufgegriffen:

- Welche Rollen kann SEO im etablierten System der Wohlfahrtsproduktion spielen, welche sind hier gemeinwohlzuträglich und innovativ, wo treten aber auch unnötige Doppelstrukturen und kontraproduktive Reibungen auf? Handelt es sich bei SEOs um „Lückenbüßer“ oder Innovationsinkubatoren? Inwiefern treten auch etablierte Wohlfahrtsproduzenten als SEO auf?

- Welche Mechanismen zeichnen SEOs aus und welche Mechanismen/Handlungsorientierungen werden von SEOs ausgefüllt? 
- Wie können SEOs produktiv in etablierte Strukturen eingepasst werden und Kooperationsstrukturen aufgebaut werden, die etablierte und neue innovative Lösungen ermöglichen? Stichworte sind hier Vernetzung und Schnittstellenmanagement.

- Wo liegen die „Einfallstore“ für soziale Unternehmen als hybride Organisationen, wie reagieren die traditionellen Anbieter sozialer Dienste, allen voran die Wohlfahrtsverbände auf diese Herausforderung?

\section{Social Entrepreneurship im deutschen Wohlfahrtsstaat: Herausforderungen und etablierte Strukturen}

Bereits seit mehr als 30 Jahren wird die Zukunftsfähigkeit der Finanzierung sozialer Dienstleistungen diskutiert - vor allem angesichts steigender Ausgaben bei gleichzeitig stagnierenden öffentlichen Einnahmen. Mittlerweile umfasst das Sozialbudget in Deutschland mehr als 750 Mrd. Euro (BMAS 2010). Über 40 Prozent des gesamten Bundeshaushaltes werden für Leistungen im Bereich Arbeit, Soziales und Gesundheit verausgabt. Ein beträchtlicher Teil sozialer Dienstleistungen wird zudem kommunal finanziert. Gemäß dem Gemeindefinanzbericht 2010 werden rund ein Viertel der kommunalen Ausgaben direkt für soziale Leistungen aufgewendet. Dieser Anteil stieg in den vergangenen Jahren kontinuierlich an und hat in der Summe 2009 erstmals die 40 Milliardengrenze überschritten (Anton/Diemert 2010: 11). Dabei sind die Aufwendungen für kommunales Personal und Sachmittel im Bereich der Sozialverwaltung und der sozialen Dienste noch nicht enthalten. Insbesondere für die Kommunen stellt sich das Problem, dass sie über ihre Einnahmen nur bedingt selbst bestimmen können und steigenden Gestaltungsaufgaben nur stagnierende Gestaltungsmittel gegenüberstehen (Grohs 2010b).

In den kommenden Jahren wird sich die Situation aufgrund sich bereits abzeichnender demographischer und sozialstruktureller Entwicklungstrends eher noch verschärfen. Weitgehend herrscht Einigkeit darüber, dass in Zukunft von steigenden Bedarfen im Bereich sozialer Dienstleistungen auszugehen ist (Evers et al. 2011). Insbesondere der demographische Wandel mit einer steigenden Zahl von älteren nicht (mehr) erwerbstätigen Menschen und Hochaltrigen wird zu wachsenden Ausgaben in den Bereichen Gesundheitsversorgung und Pflege führen (vgl. Heinze/Naegele 2010). Hinzu kommen sozialstrukturelle Veränderungen (mehr Ein- bzw. Kein-Kind-Familien, steigende Scheidungszahlen sowie eine steigende Frauenerwerbstätigkeit), die gekoppelt mit erhöhten Mobilitäts- 
anforderungen auf den Arbeitsmärkten dazu führen werden, dass sich potenzielle (und heute im Bereich der Pflege immer noch zentrale) familiäre Hilfen eher reduzieren werden (Schneiders 2010: 137ff).

Im Bereich der Kinder- und Jugendhilfe sieht die Entwicklung anders aus. Die rückläufigen Kinderzahlen führen hier z.T. zur Unterauslastung der Infrastrukturen, die allerdings durch den Ausbau der Tagesbetreuung für Unter-Dreijährige und die zunehmende Frauenerwerbstätigkeit teilweise kompensiert werden. Gerade in geburtenschwachen Regionen der neuen Bundesländer ist aber tendenziell eine Überversorgung zu verzeichnen. Während die Bedarfe in der Kinderbetreuung in Zukunft in toto eher stabil bleiben, ist im Bereich der Jugendhilfe mit ihren ambulanten und stationären Hilfestrukturen trotz der rückläufigen Kinderzahlen eine verstärkte Inanspruchnahme zu verzeichnen. Seit Einführung des Kinder- und Jugendhilfegesetzes ist hier eine nominale Verdopplung des Ausgabenvolumens festzustellen (Grohs 2010a: 145). Der Wandel von Familienstrukturen, wie die zunehmende Zahl an Alleinerziehenden und so genannter Patchwork-Familien, bringt eine größere Heterogenität in die Lebensverläufe junger Menschen, die mitunter in größeren Betreuungsbedarfen münden. Hinzu kommen auch hier abnehmende familiale Unterstützungsstrukturen. Diese Entwicklungen führen zu einem höheren Bedarf an individueller Förderung, der an die Kommunen als verantwortlichem Träger der Kinder- und Jugendhilfe herangetragen wird.

Die öffentliche Hand reagierte auf die anwachsenden Aufgaben mit der Implementation neuer Steuerungsformen, die unter den Stichworten „Managerialisierung" und „Ökonomisierung" subsumiert werden können (Evers/Heinze 2008; Heinze 2009: 61ff). Diese zielen weniger auf einen Abbau von Leistungen, als einen effizienteren und wirkungsvolleren Einsatz der zur Verfügung stehenden Ressourcen und die Aktivierung von stärkerem Wettbewerb um Qualität und Leistung ab - auch mit privaten Akteuren und social entrepreneurs. Im Bereich der Sozialen Dienste führte dies zur Übertragung betriebswirtschaftlicher Instrumente und Rationalitätsprinzipien auf traditionell unternehmensfern konstituierte Organisationen. Bedingt durch die traditionell duale Aufspaltung der sozialen Dienste in öffentliche und freie Träger findet sich der Managementdiskurs in Deutschland seit Beginn der 1990er Jahre in zwei wesentlichen Spielarten: Für den öffentlichen Bereich war das Neue Steuerungsmodell (NSM) dominierender Trend in der Übertragung betriebswirtschaftlicher Prinzipien auf die öffentlich getragenen sozialen Dienste (Bogumil et al. 2007). Im Bereich der freien Träger dominierte der ,Sozialmanagement'-Begriff die ähnlich gelagerten Umgestaltungsdiskussionen [siehe hierzu den Beitrag von Gergs]. Im Schnitt- 
stellenbereich zwischen öffentlichen Trägern (als „Auftraggebern“) und freien Trägern (als „Auftragnehmern“) wurde zudem Kontraktmanagement zum Bindeglied der Modernisierungsarenen (Grohs/Bogumil 2011). Damit verbunden ist die Einführung wettbewerblicher Elemente wie die Abkehr vom Kostendeckungsprinzip und die Aufhebung der Privilegierung der freigemeinnützigen Träger bzw. Anbietern von sozialen Dienstleistungen. Hierdurch wurde die Abkehr vom korporatistischen Modell der Leistungserstellung in allen einschlägigen Sozialgesetzbüchern eingeläutet.

In Deutschland wird - trotz aller Privatisierungs- und Ökonomisierungstendenzen - weiterhin ein Großteil der sozialen Dienstleistungen durch sog. „freigemeinnützige“ Träger erbracht. Diese stehen seit den 1990er Jahren allerdings aufgrund der neuen Steuerungsformen sowie innerverbandlicher Strukturprobleme unter erheblichem (Modernisierungs-)Druck (Heinze et al. 1997; Liebig 2005). Mit der Abkehr vom Kostendeckungs- hin zum Budgetprinzip, der Einführung wettbewerblicher Elemente bei der Vergabe von öffentlich finanzierten sozialen Dienstleistungen und der zumindest partiellen Gleichstellung privat-gewerblicher mit den ehemals privilegierten freigemeinnützigen Dienstleistungsanbietern werden die Wohlfahrtsverbände - als die zentralen Träger sozialer Dienstleistungen - vor erhebliche Herausforderungen gestellt.

Jahrzehntelang haben die Einrichtungen und Angebote, die direkt oder mittelbar den fünf großen Wohlfahrtsverbänden (Deutscher Caritasverband, Diakonisches Werk, Deutsches Rotes Kreuz, Arbeiterwohlfahrt sowie der Paritätische Wohlfahrtsverband) angehören, den ganz überwiegenden Anteil sozialer Dienstleistungen erbracht. Sowohl im Gesundheitssektor als auch in der Altenhilfe, der Kinder- und Jugendhilfe sowie der Behindertenhilfe verfügen die der BAGFW (Bundesarbeitsgemeinschaft der Freien Wohlfahrtspflege) angehörenden Verbände über erhebliche personelle und infrastrukturelle Kapazitäten. 2008 arbeiteten in über 100.000 Einrichtungen und ambulanten Angeboten der Wohlfahrtsverbände über 1,5 Mio. Beschäftigte, in denen fast 4 Mio. Menschen gepflegt, versorgt, betreut, aktiviert und beraten werden (BAGFW 2009). Die Wohlfahrtsverbände sind damit seit den 1970er Jahren zu einem der größten Arbeitgeber Deutschlands avanciert, der quantitativ betrachtet nicht den Vergleich mit großen internationalen Konzernen scheuen muss. Auf die neuen Steuerungsmodelle reagierten die etablierten wohlfahrtsverbandlichen Träger unterschiedlich. Das Spektrum der Reaktionen reichte von der punktuellen Einführung betriebswirtschaftlicher Instrumente (Controlling/Marketing) bis hin zur Ausgründung von eigenständigen privat-gewerblichen Service-Gesellschaften (Dahme et al. 2005; Grohs 2010a; Schneiders 2010). 
Neben Modernisierungsprozessen bei den freigemeinnützigen und einer Expansion der privat-gewerblichen Anbieter in einigen Bereichen sind im Feld der sozialen Dienstleistungen in den letzten Jahren Verknüpfungen von sozialem Engagement und unternehmerischem Handeln erkennbar, die medienwirksam unter dem Label „Social Entrepreneurship“ vermarktet werden. Die Thematisierung der Verknüpfung von unterschiedlichen Handlungsorientierungen innerhalb einer Organisation ist indes nicht neu. Das Konzept des „Dritten Sektors" greift gerade solche Organisationen auf, die sich keinem der drei Sektoren „Markt", „Staat" oder „Gemeinschaft“ zuordnen lassen (zusammenfassend Evers/Ewert 2010; Salamon/Anheier 1996).

Neu ist indes der Hype nach dem Motto „Everyone can change the world“ (Bornstein 2007; Elkington/Hartigan 2008; Bornstein/Davis 2010), mit dem ausgewählte Gründerpersönlichkeiten insbesondere von Mittlerorganisationen in Szene gesetzt werden und mittlerweile eine große Zahl von semiwissenschaftlichen Beiträgen hervorgebracht hat. Es stellt sich die Frage, ob es sich bei diesen Organisationen um eine originär neue Form der Leistungserstellung handelt, die gegenüber den etablierten Trägern über spezifische Vorteile verfügt und ob diese zu einer Lösung des Dilemmas von zunehmenden Bedarfen bei gleichzeitig stagnierenden öffentlichen Ressourcen beitragen können.

\section{Social Entrepreneurship - Begriffliche Annäherung}

Bislang hat sich in Deutschland keine einheitliche Definition des aus dem Angelsächsischen stammenden Begriffs „Social Entrepreneurship“ durchsetzen können. Neben der Abhängigkeit vom jeweiligen institutionellen Kontext variieren die Interpretationen dessen, was unter SEO zu verstehen ist, mit der wissenschaftlichen Disziplin, aber auch mit der Motivation, Message oder Handlungsorientierung der den Begriff verwendenden Autorinnen und Autoren.

Vielfach fokussiert SEO auf die Erfassung der einzelnen, förderungswürdigen social entrepreneurs als Teilausschnitt des organisationssoziologischen Phänomens SEO. Dieses Verständnis ist geprägt von einer starken Fokussierung auf einzelne Initiatoren bzw. Handelnde. Die Akteurszentrierung resultiert aus der hervorstechenden Stellung einer Einzelperson für die Organisation. Entweder hat sie die Erschließung eines neuen Handlungsfelds initiiert oder eine neue, den herausragenden Erfolg der Organisation begründende, Herangehensweise an ein gesellschaftliches Problem eingeführt. Die in diesem Sinne verstandenen social entrepreneurs finden ihre Motivation in dem Willen, eine drängende ge- 
sellschaftliche Frage zu bearbeiten. Ein solches Verständnis wird beispielsweise der Förderung mit Stipendien durch Ashoka zugrunde gelegt (Ashoka 2010). Die Risikobereitschaft begründet sich hier aus einer philanthropischen Haltung: meist ist das Engagement biographisch erklärbar. Dies mag im Hinblick auf eine Lenkung der medialen Aufmerksamkeit zweckmäßig sein, begrenzt allerdings den Untersuchungsgegenstand auf eine äußerst kleine Gruppe von Akteurinnen und Akteuren.

Ausgehend von den Teilbegriffen ,Social' und ,Entrepreneurship' umfasst social entrepreneurship in Deutschland jedoch ein sehr viel weiteres Akteurspektrum und ein breites Angebotsfeld. Als „social“ können solche „entrepreneurships" bezeichnet werden, die in zweifacher Weise gesellschaftliche Relevanz aufweisen: hinsichtlich ihres Aufgabenspektrums und ihrer Ausstrahlungskraft in die Gesellschaft. Der Tätigkeitsbereich sollte insofern Dienstleistungen umfassen, die sich an Menschen in besonderen Problemlagen bzw. mit besonderen Hilfebedarfen richten und dazu dienen, diese Problemlagen zu reduzieren und Hilfebedarfe zu befriedigen. Die den SEOs von uns unterstellte Gemeinwohlorientierung äußert sich in den realisierten Effekten und ist konstitutives Element der jeweiligen Unternehmenskultur.

Der Begriff des „Unternehmerischen“ (entrepreneurship) kann im organisationssoziologischen Sinne als innovationsorientiertes, strategisches und seine Risiken selbst verantwortendes Handeln von Organisationen verstanden werden. Unternehmerisches Handeln zeichnet sich einerseits durch seine strategische Orientierung aus: Es ist in Abgrenzung zu bürokratischem Handeln an Zielen und Ergebnissen orientiert und nicht an einer ,Abarbeitung' vorgegebener Aufgaben. Strategisches Handeln heißt auch die Nutzung von Verfahren und Instrumenten, die es ermöglichen, rechtzeitig auf ein Nichterreichen der Ziele zu reagieren (Management). Zweites Kennzeichen unternehmerischen Handelns ist die Innovationsorientierung. Ziel dieser Orientierung ist es, neue Handlungsfelder zu entdecken und zu bearbeiten oder bekannte Felder (durch Evaluation) neu anzugehen. Drittes Kennzeichen ist die Verantwortlichkeit für mögliches Scheitern, also eine Internalisierung des eigenen Geschäftsrisikos.

Aus organisationssoziologischer Perspektive handelt es sich bei SEOs um hybride Organisationen (Evers et al. 2002; Priddat 2009a; Billis 2010), die verschiedene Handlungsorientierungen integrieren. Demzufolge sind auch verschiedene Varianten vorhanden. SEO bewegt sich dabei zwischen den Polen einer marktgetriebenen - auch gewinnorientierten - Orientierung, einer an gemeinschaftlichen Werten orientierten Perspektive und einer auf das ,große Ganze“ gerichteten „staatsorientierten“ bürokratischen Rationalität. SEOs weisen - 
so die These - eine besondere Form des Mischungsverhältnisses dieser drei Handlungsorientierungen auf. „Hybridity is not therefore any mixture of features from different sectors, but according to this view, is about fundamental and distinctly different governance and operational principles in each sector" (Billis 2010: 3; vgl. auch Brandsen et al. 2010).

SEOs können daher, müssen aber keine non-profit-Organisationen im Sinne des „Dritten Sektors“ sein. Denn entgegen der von Salamon/Anheier (1996: 125ff) entwickelten Kriterien für Dritte Sektor-Organisationen weisen sie zumindest zeitweise keine formale Struktur auf und können neben einer Gemeinwohl- auch eine Profitorientierung aufweisen. ${ }^{1}$ SEO kann nicht nur in Bezug auf die Integration verschiedener Handlungsorientierungen als hybrid bezeichnet werden, auch die Organisationsstruktur kann keiner Rechtsform eindeutig zugeordnet werden. So ist SEO nach der oben skizzierten Definition sowohl als Einzelunternehmen, Stiftung, gemeinnütziger Verein/Verband als auch in Form von Public Private Partnership (PPP) denkbar. Das Spektrum reicht dabei von der unternehmensnahen Stiftung mit einem Budget von mehreren Mio. Euro über den Mitarbeiterstab eines Konzerns bis hin zur Initiative Einzelner, die sich eines von ihnen als drängend empfundenen sozialen Problems annehmen.

Im Umfeld dieses SEO-Phänomens haben sich eine Reihe von weiteren Begriffen etabliert, die Schnittstellen mit den hier im Mittelpunkt stehenden Organisationen aufweisen, definitorisch von ihnen jedoch abzugrenzen sind, wie „Soziale Unternehmen“, aber auch die inzwischen breite Diskussion um „Corporate Social Responsibility“ (CSR) und „Corporate Citizenship“ (BackhausMaul et al. 2010). So steht anders als bei „Sozialen Unternehmen“ bei SEOs keine arbeitsmarktpolitische Motivation im Vordergrund. Mit dem Begriff „Soziale Unternehmen" werden bisher vielmehr Organisationen bezeichnet, die Problemgruppen des Arbeitsmarktes eine Beschäftigungsperspektive bieten (Defourny/Nyssens 2010). Soziale Unternehmen bewegen sich am Markt insofern, als sie Produkte und Dienstleistungen anbieten, aber auch sozialpädagogische bzw. weiterbildende Elemente in den laufenden Betrieb integrieren. Die Lohnkosten für die Beschäftigten sowie für den Overhead aus fach- und sozialpädagogischem Personal werden nur zum Teil aus der Vermarktung der Produkte und Dienstleistungen bestritten, i.d.R. werden die Sozialen Unternehmen im Rahmen arbeitsmarktpolitischer Förderprogramme durch die öffentliche Hand subventioniert.

1 Dabei ist darauf hinzuweisen, dass die der John-Hopkins-Studie zugrunde liegende Definition von Dritte Sektor Organisationen als Residualkategorie (weder eindeutig „Markt“ noch eindeutig „Staat") der tatsächlichen Struktur und Ausgestaltung der unter diesem Label zusammengefassten Organisationen immer weniger gerecht wird (zu UK und USA vgl. Billis 2010). 
Analogien zwischen social entrepreneurship und dem Begriff „Corporate Social Responsibility“ können sich hinsichtlich der strategischen Vorgehensweise, den Handlungsfeldern und der praktischen Ausgestaltung von Konzepten zeigen. Da CSR jedoch hauptsächlich von privatwirtschaftlichen Organisationen mit Renditeorientierung ausgeht, muss eine Unterscheidung hinsichtlich des Aktionsrahmens, der Initiatorinnen bzw. Initiatoren und der übergeordneten Zielorientierung getroffen werden.

Unternehmerische CSR kann zwar mit einer Vielzahl von Faktoren wie Unternehmenskultur, Reputation, Renditesteigerung, Risikosicherung, gesellschaftliche Legitimation sowie der Notwendigkeit, Humanvermögen und -kapital aufzubauen, begründet werden. Dennoch ist die strategische Orientierung von CSR untrennbar mit den übergeordneten Unternehmenszielen verknüpft, wodurch sie weniger auf eine aktive Gestaltung der Gesellschaft und deren Rahmenbedingungen fokussiert, als vielmehr eine Antizipation und Reaktion auf Umweltbelange anstrebt. CSR dient demnach der Verbesserung und Anpassung bestehender Strukturen des unternehmerischen Handelns und ist daher systemimmanent. SEO weist dagegen eine größere Heterogenität auf und zeichnet sich durch Innovativität, Hybridität und transzendente Lösungsansätze aus, die mit unternehmerischen Mitteln erreicht werden sollen, jedoch nicht zwangsläufig renditeorientiert sind.

\begin{tabular}{|c|c|c|}
\hline \multirow{3}{*}{ 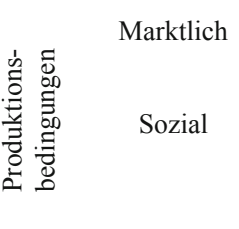 } & Social Entrepreneurship & $\begin{array}{c}\text { Privat-gewerbliches } \\
\text { Unternehmen }\end{array}$ \\
\hline & Non-Profit-Organisation & $\begin{array}{c}\text { Social Enterprise } \\
\text { (Soziales Unternehmen) }\end{array}$ \\
\hline & Sozial & Marktlich \\
\hline
\end{tabular}

Abbildung 1: Social Entrepreneurship im Kontext

Daten zur Zahl der Organisationen und ihrer Beschäftigten sowie den von ihnen bearbeiteten Tätigkeitsfeldern sind bislang jenseits der eindeutig dem privatgewerblichen Unternehmen zuzuordnenden Organisationen, für den auch amtliche Statistiken vorliegen, nur in unsystematischer Form vorhanden. Dies ist vor allem auf die Hybridität der Organisationen zurückzuführen, die eine eindeutige Zuordnung erschwert. Der hier interessierende SEO-Sektor wird in Deutschland aber um ein Vielfaches größer sein, als die bei Ashoka (www.ashoka.org) und der Schwab Foundation (www.schwabfound.org) akkreditierten 27 bzw. 6 „Fel- 
lows“ bzw. „Social Entrepreneurs“. Im Folgenden explizieren wir, warum insbesondere der soziale Dienstleistungssektor von Organisationen, die dem Typ SEO zuzuordnen sind, geprägt ist. ${ }^{2}$

\section{Produktionsbedingungen im sozialen Dienstleistungssektor}

Die Produktion sozialer Dienstleistungen ist ein komplexer Prozess. Zur Komplexität tragen sowohl die Spezifika der Dienstleistungen selbst (wie z.B. das uno actu-Prinzip bzw. die Koproduktion durch die Klienten und die schwierige Qualitätsbeurteilung) als auch die jeweiligen Austauschprozesse zwischen Konsumierenden und Produzierenden bei (Evers et al. 2011). Zwischen ihnen besteht eine Asymmetrie bezüglich des Wissens um die Notwendigkeit einerseits und die qualitative Ausgestaltung des Dienstleistungsprozesses andererseits. Konsumentensouveränität ist nicht nur hinsichtlich des Umfangs und der Art der Dienstleistungen limitiert, sondern auch wegen der häufig eingeschränkten $\mathrm{Zu}$ gangsmöglichkeiten und/oder individueller Fähigkeiten. So wird von einem delinquenten Jugendlichen der Bedarf an Hilfe nicht wahrgenommen und auch die angebotenen sozialen Dienstleistungen werden von ihm weder ausgewählt noch sind sie in ihrer Qualität von ihm beurteilbar. Die Diskussion um Qualitäts- und Wirkungsmessung ist in diesem Feld zwar umfangreich, jedoch noch weit davon entfernt, etablierte Standards gefunden zu haben.

In anderen Hilfefeldern wie der Altenpflege wurden in den letzten Jahren eine Reihe von Steuerungsmaßnahmen ergriffen, um die Transparenz im Sektor zu erhöhen (Qualitätsberichte, Pflegenoten). Hinzu kommt eine hohe Regulationstiefe, die den Spezifika geschuldet ist, aber auch damit zusammenhängt, dass ein Großteil der sozialen Dienstleistungen eben nicht im Rahmen von Marktprozessen zwischen Kunden und Produzenten (Dienstleistung gegen Geld), sondern in einem Dreiecksverhältnis zwischen Kostenträger, Kunde und Leistungserbringer erbracht werden. Dabei setzte seit Mitte der 1990er Jahre (Einführung der Pflegeversicherung, Reform der Arbeitsverwaltung, Kommunalisierung der Eingliederungshilfe etc.) eine Differenzierung auf Seiten der öffentlichen Kostenträger ein, die das Feld für Leistungsempfänger und -anbieter unübersichtlicher gestaltet.

2 Vgl. zum Versuch, einer systematischen Erfassung der Organisationen, die der „Zivilgesellschaft" zuzuordnen sind, das beim Deutschen Stifterverband (www.stifterverband.de) angesiedelte Projekt „Zivilgesellschaft in Zahlen“. 
Für soziale Aufgabenfelder, die „quer“ zu den etablierten Strukturen liegen und bspw. Nischen bearbeiten, ist es schwierig, adäquate Finanzierungsstrukturen aufzubauen, da ein hoher Regulierungsgrad entlang der Säulen der Sozialgesetzgebung existiert, der häufig mit den etablierten Strukturen der Wohlfahrtsproduktion kongruent ist. Anbieter, die dem SEO-Bereich zugerechnet werden können, stoßen in dieser Landschaft auf zahlreiche institutionelle Hürden: Für neue Angebote existiert häufig keine klare öffentliche Finanzierungsverantwortung für innovative Aufgaben, so dass ein Lobbying in eigener Sache notwendig wird. Im Feld der etablierten Aufgaben finden sich häufig geschlossene Märkte, da weiterhin bestehende korporatistische Routinen überwiegen und eine enge Verwobenheit zwischen Fachverwaltungen als Kostenträgern und etablierten Anbietern aus der Verbändelandschaft zu beobachten ist.

Innovationsstrategien, die sich an einer qualitativen Verbesserung bestehender Angebote orientieren, treffen auf eine Welt, in der häufig eindeutige Qualitätsstandards fehlen. Öffentliche Kostenträger bringen neuen Spielern erhebliches Misstrauen entgegen. Anstelle tatsächlicher Qualitätspolitik wird häufig eine Art „Qualitätsmanagement by Rumour“ (Grohs 2010a), also eine Orientierung am allgemeinen Ruf der Einrichtungen betrieben. Auch wenn mittlerweile auf dem Rechtsweg eine Reihe von korporatistischen Residuen beseitigt wurden - bspw. die Abschaffung der kommunale Bedarfsbestätigung als Voraussetzung für die Gewährung öffentlicher Mittel im stationären Altenpflegesektor (Schneiders 2010: 224f) - diskriminieren rechtliche Regulierungen noch immer in vielen Feldern neue private Anbieter (vgl. z.B. die Regelungen zum Begriff des Freien Trägers im SGB VIII) im Marktzutritt und auch bei der Beteiligung an Entscheidungsgremien wie dem Jugendhilfeausschuss. Es bleibt abzuwarten, inwieweit sich hier eine zunehmende Justizialisierung politischer Entscheidungen entwickeln wird.

\section{Social Entrepreneurship in zwei ausgewählten Sektoren: Altenpflege und Kinder- und Jugendhilfe}

Die skizzierten Produktionsbedingungen haben maßgeblich dazu beigetragen, dass bis in die 1990er Jahre hinein der ganz überwiegende Teil sozialer Dienstleistungen von freigemeinnützigen Anbietern, in der Hauptsache von den Wohlfahrtsverbänden bzw. den ihnen angehörigen Trägern, angeboten wurden. Als weitere Akteure waren in der Altenpflege private Anbieter, in der Kinder- und Jugendhilfe öffentliche Träger relevante Anbieter. 
In der Altenpflege sind seit der Einführung der Pflegeversicherung Mitte der 1990er Jahre zum Teil erhebliche Veränderungen in der Trägerstruktur erkennbar (Schneiders 2010). Mit der Aufhebung der Privilegierung der freigemeinnützigen Anbieter sind vermehrt privat-gewerbliche Anbieter auf dem neu formierten „Pflegemarkt“ aktiv. Aus den Daten der Pflegestatistik für das Jahr 2007 (Statistisches Bundesamt 2008) wird zunächst deutlich, dass sich die Mehrheit der Pflegeheime (ca. 55 Prozent) weiterhin in Trägerschaft von freigemeinnützigen Organisationen befindet. Privat-gewerbliche Anbieter machen einen Teil von ca. 39 Prozent aus. In öffentlicher bzw. kommunaler Trägerschaft befinden sich 6 Prozent aller Einrichtungen. Aufgrund der erheblichen Heterogenität der privat-gewerblichen Träger mit einer Vielzahl von kleineren privaten Pflegeheimen (ca. 60 Prozent verfügen über weniger als 50 Plätze) liegt die durchschnittliche Platzzahl in privaten Einrichtungen mit 63 deutlich unter der durchschnittlichen Einrichtungsgröße der freigemeinnützigen Anbieter (77 Plätze/Einrichtung) bzw. der von der öffentlichen Hand getragenen Pflegeheime (85 Plätze/Einrichtung). Im Vergleich mit den Strukturdaten aus 1999 (dem ersten Berichtsjahr zur Pflegeversicherung) werden folgende Trends deutlich. Die Zahl der Pflegeheime insgesamt ist um ca. 17 Prozent gestiegen. Dieses Wachstum ist insbesondere auf eine zunehmende Zahl von Einrichtungen in privat-gewerblicher ( +40 Prozent) und in freigemeinnütziger Trägerschaft (+21 Prozent) zurückzuführen. Die Zahl der Pflegeheime in öffentlicher Trägerschaft ist hingegen rückläufig ( -15 Prozent).

Im regionalen Vergleich fällt auf, dass die privat-gewerblichen Träger insbesondere in den Bundesländern, in denen sie bereits über hohe Marktanteile verfügten, ihre Position weiter ausbauen konnten. So sind in Schleswig-Holstein mittlerweile über 60 Prozent der Plätze in privat-gewerblicher Trägerschaft und auch in Niedersachsen haben die privat-gewerblichen Träger die freigemeinnützigen als „Marktführer“ abgelöst. Die freigemeinnützigen Anbieter büßen auch in Brandenburg an Bedeutung ein. Öffentliche Träger verfügen nur noch in Hamburg, Baden-Württemberg und Bayern über nennenswerte Anteile, in den übrigen Ländern wurden die Kapazitäten deutlich abgebaut.

Die Daten zeigen, dass sich in den letzten Jahren - mit stärkerer Dynamik seit Einführung der Pflegeversicherung im Jahr 1995 - privat-gewerbliche Anbieter im Sektor der stationären Pflege etabliert haben. Zu diesen Anbietern gehören sehr kleine und mittelständische Unternehmen sowie einige größere Anbieter, die zum Teil börsennotiert sind. In der ambulanten Pflege ist das Wachstum insgesamt fast ausschließlich auf die Expansion privat-gewerblicher Anbieter (+55 Prozent) zurückzuführen. 


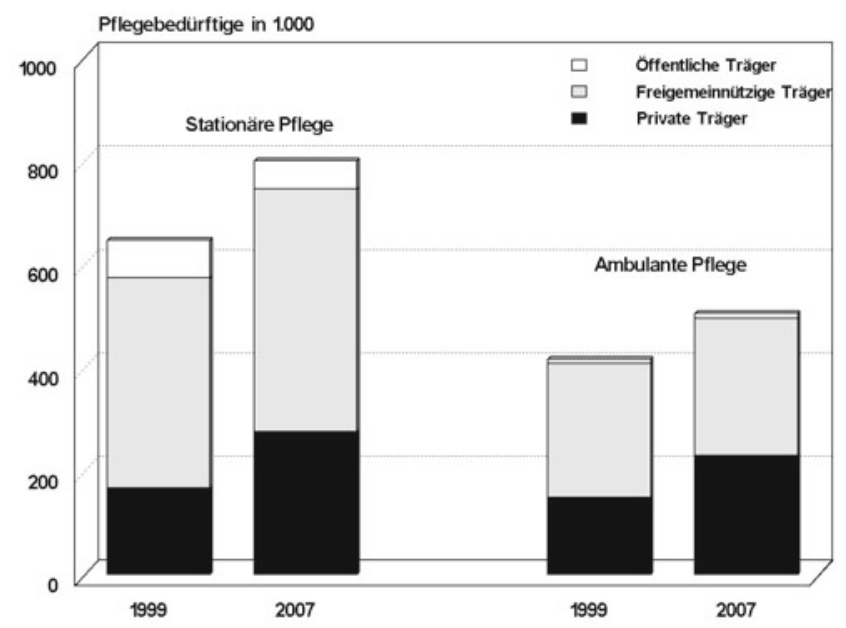

Abbildung 2: Trägerstrukturen in der Altenpflege (Statistisches Bundesamt 2008; eig. Darstellung)

Die Betrachtung auf der Basis statistischer Rahmendaten wird der hier verfolgten Fragestellung nach den Organisationsformen jedoch nicht gerecht. Bei genauerem Hinsehen ist nämlich feststellbar, dass in einigen dem freigemeinnützigen Sektor zugeordneten Einrichtungen bzw. ambulanten Angeboten durchaus auch unternehmerische bzw. marktgetriebene Elemente erkennbar sind. So bezeichnet sich bspw. die CBT Köln (www.cbt-gmbh.de) als sozialwirtschaftliches Unternehmen, das eine Vielzahl von unternehmerischen Elementen integriert hat - ähnlich wie die Stiftung Liebenau (www.stiftung-liebenau.de) in BadenWürttemberg.

Hinzu kommen immer mehr freigemeinnützige Anbieter, die (privat-gewerbliche) Tochtergesellschaften ausgründen, u.a. um so die Tarifverträge der Wohlfahrtsverbände auszuhebeln. Auf der anderen Seite sind nicht alle privatgewerblichen Anbieter ausschließlich renditeorientiert. Auch hier finden sich insbesondere bei den kleinen und mittelständischen Unternehmen - viele Organisationen, deren Wirtschaftsbetrieb auf Kostendeckung ausgerichtet ist. Die Rechtsform einer Organisation ist immer weniger mit der Handlungsorientierung gleichzusetzen. Der Status „freigemeinnützig“ ist also nicht mehr unmittelbar mit „gemeinwohlorientiert“ gleichzusetzen oder anderweitig positiv normativ aufgeladen (hierzu auch Hopt et al. 2005; BAGFW 2010; Wendt 2010). 
Auch wenn weiterhin „,korporatistische Einsprengsel“ unübersehbar sind (bspw. lokale Pflegekonferenzen, die wohlfahrtsverbandlich dominiert werden), zeigt sich, dass sich marktförmige Koordinationsmechanismen (teilweise in Form der Justizialisierung) zunehmend durchsetzen (Schneiders 2010).

Ein wesentlich anderes Bild zeigt sich im Feld der Kinder- und Jugendhil$f e$, die einerseits die Kindertagesbetreuung, andererseits Angebote der stationären und ambulanten Jugendhilfe umfasst. Auch hier wurde gesetzgeberisch eine Reihe von Maßnahmen vorgenommen, um das Akteurspektrum zu erweitern. Genannt seien hier nur die Einführung prospektiver Leistungsvereinbarungen bzw. Leistungsentgelten sowie der Einführung von Qualitätssicherungs- und Leistungsstandards nach den $\S \S 78 \mathrm{a}-\mathrm{g}$ SGB VIII und die Aufhebung des bedingten Vorrangs freigemeinnütziger Träger (Grohs 2010a: 70ff). Diese haben an den Trägerstrukturen bislang jedoch kaum etwas geändert: Betrachten wir die Trägerschaft der Einrichtungen, die öffentlich mitfinanzierte Angebote erbringen, so fällt für Westdeutschland eine überraschende Kontinuität im Zeitverlauf auf (Abbildung 3).

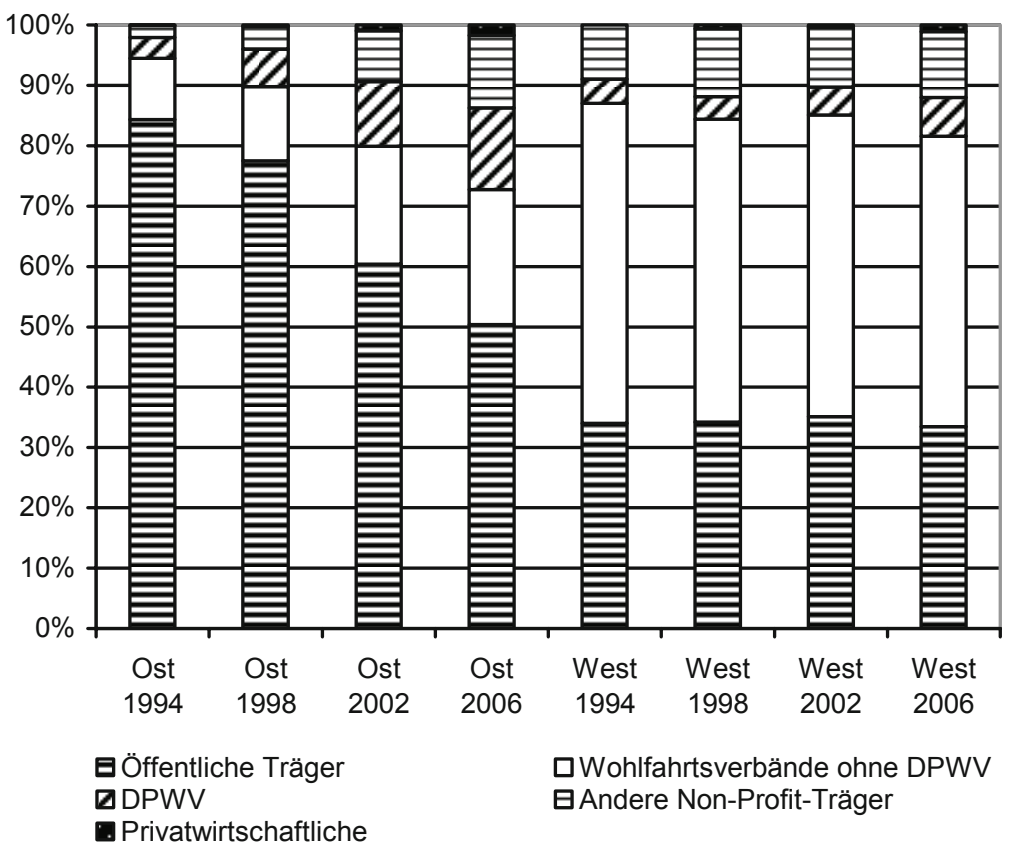

Abbildung 3: Wandel der Trägerstrukturen Kindertagesbetreuung (Grohs 2010a: 147) 
Sowohl im Bereich der Kindertagesbetreuung wie im Bereich der Jugendhilfe sind in den alten Bundesländern über den Beobachtungszeitraum kaum Veränderungen in den Anteilen der einzelnen Trägergruppen festzustellen. Trotz Verschiebungen im Aufgabenportfolio (Ausbau Betreuung Unter-Dreijährige, Umstellung von stationärer zu ambulanter Unterbringung etc.) ist es den bisherigen Trägerensembles offensichtlich gelungen, ihre jeweiligen Anteile zu bewahren. Eine Ausnahme stellt das relativ neue Feld der ,Kleinsteinrichtungen der stationären Erziehungshilfe" dar, ein hoch spezialisiertes Segment und der einzige Bereich, in dem privatwirtschaftliche Anbieter einen nennenswerten Anteil stellen (rund 30 Prozent im Jahr 2006).

Für die neuen Bundesländer ist im Zeitverlauf in erster Linie eine „Entkommunalisierung" kennzeichnend. Von der aus DDR-Zeiten übernommenen kommunalen Eigenproduktion entwickeln sich die ostdeutschen Wohlfahrtsarrangements hin zu einer durchaus mit dem Westen vergleichbaren Gewährleistungsverwaltung. In den alten Bundesländern bleiben zumindest nach der öffentlichen Statistik die öffentlichen Anteile weitgehend konstant. Generell konnten sich die westdeutschen Wohlfahrtsverbände auch in Ostdeutschland ein beachtliches Terrain sichern - gegeben der Tatsache, dass sie nach 1990 ihre gesamte Infrastruktur neu aufbauen mussten (Angerhausen et al. 1998). Der DPWV hat in Ostdeutschland durchweg größere Anteile, was zum einen auf durch das Aufgehen der zu DDR-Zeiten quasistaatlichen Einrichtungen der „Volkssolidarität“ im DPWV, andererseits das Eintreten zahlreicherer kleinerer Projekte in den DPWV zurückzuführen ist. Insgesamt zeichnen sich die ostdeutschen Strukturen dennoch durch eine größere Pluralität, allerdings einen noch geringeren Anteil privatgewerblicher Anbieter aus.

Trotz einzelner Nischen kann also konstatiert werden, dass zumindest für Westdeutschland gemessen an der Trägerschaft von einer Auflösung des „Wohlfahrtskorporatismus" nicht die Rede sein kann: Für den Osten ist eine Angleichung der Strukturen an Westdeutschland zu beobachten, jedoch wird diese Tendenz durch einen höheren Anteil „,bürgerschaftlich“ getragener Einrichtungen außerhalb der Wohlfahrtsverbände ergänzt. Privatwirtschaftliche Akteure und insbesondere SEOs als Nischenunternehmer spielen nur eine marginale Rolle. Wirkliche Wandlungstendenzen scheinen sich nur durch externen Druck und eher hierarchische Implementationsstrukturen durchsetzen zu lassen. Die etablierten Arrangements scheinen sich aus der Sicht der Akteure vor Ort bewährt zu haben. Häufig stehen Alternativen zu etablierten Akteuren auch schlicht nicht zur Verfügung. Die Positions- und Zugangsregeln bleiben weitgehend in Kraft: Weiterhin findet sich eine starke Verflechtung der Verbände mit der Sozialadministration und den örtlichen Parteien (Grohs 2010a). 
Die in diesem Abschnitt präsentierten Daten können freilich nur den IstZustand abbilden. Über das Potential, dass SEO entfalten kann, ist damit zunächst noch wenig ausgesagt. Gleichwohl lassen sich aus den beiden betrachteten Feldern Rahmenbedingungen identifizieren, die eine Etablierung von SEO als einer Erscheinung, die über Einzelfälle hinausgeht, erschweren.

\section{Fazit und Ausblick: Social Entrepreneurship als Lösung der wohlfahrtsstaatlichen Probleme?}

Das social entrepreneurship-Konzept weist aufgrund seines (scheinbar) visionären Charakters eine hohe Attraktivität auf. Ein Ausweg aus dem Dilemma der wachsenden sozialen Aufgaben bei stagnierenden öffentlichen Finanzen scheint gefunden. Zudem zeichnet sich ein qualitativer Mehrwert durch das persönliche Engagement der „Unternehmerinnen und Unternehmer“ im Bereich der sozialen Dienstleistungen ab. Die Ausführungen zu den Begrifflichkeiten sowie die Darstellung der „Produktionsbedingungen“ im deutschen sozialen Dienstleistungssektor haben jedoch gezeigt, dass die Übertragung eines Modells aus dem angelsächsischen Raum auf die deutsche „Wohlfahrtsstaatswirklichkeit" nur begrenzt möglich ist. Vielmehr müssen zunächst Begrifflichkeiten und Konzepte an das jeweilige wohlfahrtsstaatliche Regime angepasst und die jeweiligen institutionellen Kontexte einbezogen werden. Eine Theoriebildung der hybriden Organisationen für Deutschland steht vor diesem Hintergrund noch aus. Nur so könnte überprüft werden, inwiefern es sich bei SEO um ein konsistentes Konzept handelt, mit dem die neuen hybriden Strukturen zwischen Markt, Staat und Gemeinschaft besser als mit dem bereits vorhandenen „Dritter Sektor“-Modell erfasst werden können und ggf. den Charakter eines Leitbildes für die Modernisierung der etablierten wohlfahrtsstaatlichen bzw. sozialpolitischen Strukturen entwickeln kann.

Ebenfalls steht eine empirische Überprüfung bezüglich der Wirkungen von SEO noch aus. Ein von der Stiftung Mercator geförderter Forscherverbund ${ }^{3}$ widmet sich genau diesen Fragestellungen. In dem von uns verantworteten stark empirisch angelegten - Projekt „Social Entrepreneurship im etablierten Wohlfahrtsstaat. Lückenbüßer oder Innovationsinkubator" wird anhand von zwei Sektoren (kultursensible Altenpflege und -hilfe sowie Förderung der Bildung von Kindern mit Migrationshintergrund) ermittelt, in welchem Umfang

3 www.stiftung-mercator.de/kompetenzzentren/wissenschaft/social-entrepreneurship.html (letzter Aufruf: 14.11.2010). 
SEO-Strukturen im oben definierten Sinne bereits erkennbar sind, welche Ausstrahlungskraft auf etablierte Trägerstrukturen Projekte aufweisen und inwiefern die aktuellen politischen und rechtlichen Rahmenbedingungen fördernd oder eher hemmend auf die Entwicklung von SEO wirken.

\section{Quellenverzeichnis}

Angerhausen, Susanne/Backhaus-Maul, Holger/Offe, Claus/Olk, Thomas/Schiebel, Martina (1998): Überholen ohne Einzuholen. Freie Wohlfahrtspflege in Ostdeutschland. Opladen.

Anton, Stefan/Diemert, Dörte (2010): Kommunale Finanzen: Kein Licht am Ende des Tunnels! Gemeindefinanzbericht 2010 im Detail. In: Der Städtetag 2010/5: 11-85.

Ashoka (2010): Jahresbericht 2009, Berlin. http://germany.ashoka.org/sites/germany.ashoka.org/ files/Ashoka_JB_2009\%20final\%20Web.pdf (letzter Aufruf: 2.11.2010).

Backhaus-Maul, Holger/Biermann, Christiane/Nährlich, Stefan/Polterauer, Judith (Hrsg.) (2010): Corporate Citizenship in Deutschland: Bilanz und Perspektiven. 2. erw. Auflage. Wiesbaden.

BAGFW - Bundesarbeitsgemeinschaft der Freien Wohlfahrtspflege (Hrsg.) (2009): Gesamtstatistik 2008. Berlin.

BAGFW - Bundesarbeitsgemeinschaft der Freien Wohlfahrtspflege (Hrsg.) (2010): Sozialwirtschaft - mehr als Wirtschaft? Baden-Baden.

Benz, Arthur et al. (Hrsg.) (2007): Handbuch Governance. Theoretische Grundlagen und empirische Anwendungsfelder. Wiesbaden.

Billis, David (Hrsg.) (2010): Hybrid organizations and the third sector. Challenges for practice, theory and policy. Hamshire.

BMAS (2010): Sozialbudget 2009. Berlin. www.bmas.de/portal/46628/property=pdf/a230-09_sozialbudget_2009.pdf (letzter Aufruf: 14.11.2010).

Bogumil, Jörg/Grohs, Stephan/Kuhlmann, Sabine/Ohm, Anna K. (2007): Zehn Jahre Neues Steuerungsmodell - eine Bilanz kommunaler Verwaltungsmodernisierung. Berlin.

Bornstein, David (2007) How to change the world: Social entrepreneurs and the power of new ideas. New York.

Bornstein, David/Davis, Susan (2010): Social entrepreneurship: What everyone needs to know. New York.

Brandsen, Taco/Dekker, Paul/Evers, Adalbert (Hrsg.) (2010): Civicness in the governance and delivery of social services. Baden-Baden.

Dahme, Hans-Jürgen/Kühnlein, Gertrud/Wohlfahrt, Norbert/Burmester, Monika (2005): Zwischen Wettbewerb und Subsidiarität. Wohlfahrtsverbände unterwegs in die Sozialwirtschaft. Berlin.

Defourny, Jacques/Nyssens, Marthe (2010): Conceptions of social enterprise and social entrepreneurship in Europe and the United States: Convergences and divergences. In: Journal of Social Entrepreneurship 1/2010/1: 32-53.

Elkington, John/Hartigan Pamela (2008): The power of unreasonable people: How social entrepreneurs create markets that change the world. Boston.

Evers, Adalbert/Heinze, Rolf. G. (Hrsg.) (2008): Sozialpolitik. Ökonomisierung und Entgrenzung. Wiesbaden. 
Evers, Adalbert/Ewert, Benjamin (2010): Hybride Organisationen im Bereich sozialer Dienste. Ein Konzept, sein Hintergrund und seine Implikationen. In: Klatetzki (2009): 103-128.

Evers, Adalbert/Heinze, Rolf G./Olk, Thomas (Hrsg.) (2011): Handbuch Soziale Dienste. Wiesbaden.

Evers, Adalbert/Rauch, Ulrich/Stitz, Uta (2002): Von öffentlichen Einrichtungen zu sozialen Unternehmen. Berlin.

Grohs, Stephan (2010a): Modernisierung kommunaler Sozialpolitik. Anpassungsstrategien im Wohlfahrtskorporatismus. Wiesbaden.

Grohs, Stephan (2010b): Kommunale Sozialpolitik in der Haushaltskrise: Handlungsfelder und Handlungsstrategien. Expertise für die Hans Böckler-Stiftung.

Grohs, Stephan/Bogumil, Jörg (2011): Management sozialer Dienste. In: Evers et al. (2011): 219-314.

Heinze, Rolf G. (2009): Rückkehr des Staates? Politische Handlungsmöglichkeiten in unsicheren Zeiten. Wiesbaden.

Heinze, Rolf G./Naegele, Gerhard (Hrsg.) (2010): Einblick in die Zukunft. Gesellschaftlicher Wandel und Zukunft des Alterns. Berlin/Münster.

Heinze, Rolf G./Schmid, Josef/Strünck, Christoph (1997): Zur politischen Ökonomie der sozialen Dienstleistungsproduktion. Der Wandel der Wohlfahrtsverbände und die Konjunkturen der Theoriebildung. In: Kölner Zeitschrift für Soziologie und Sozialpsychologie 1997: 242-271.

Hopt, Klaus J./v. Hippel, Thomas/Walz, W. Rainer (Hrsg.) (2005): Nonprofit-Organisationen in Recht, Wirtschaft und Gesellschaft. Theorien - Analysen - Corporate Governance. Tübingen.

Klatetzki, Thomas (Hrsg.) (2010): Soziale personenbezogene Dienstleistungsorganisationen. Soziologische Perspektiven. Wiesbaden.

Liebig, Reinhard (2005): Wohlfahrtsverbände im Ökonomisierungsdilemma. Freiburg.

Priddat, Birger (2009a): Social Entrepreneurship - Eine theoretische Einschätzung, in: Priddat (2009b): 231-243.

Priddat, Birger (Hrsg.) (2009b): Nonprofit-Wirtschaft. Zwischen Staat, Wirtschaft und Gesellschaft. Neuere Einsichten. Marburg.

Salamon, Lester M./Anheier, Helmut K. (1996): The emerging nonprofit sector. An overview. Manchester.

Schmid, Josef (2010): Wohlfahrtsstaaten im Vergleich. Soziale Sicherung in Europa: Organisation, Finanzierung, Leistungen und Probleme. 3. Auflage. Wiesbaden.

Schneiders, Katrin (2010): Vom Altenheim zum Seniorenservice. Institutioneller Wandel und Akteurkonstellationen im sozialen Dienstleistungssektor. Baden-Baden.

Statistisches Bundesamt (2008): Pflegestatistik 2007. Pflege im Rahmen der Pflegeversicherung. Deutschlandergebnisse. Wiesbaden.

Wendt, Wolf Rainer (Hrsg.) (2010): Wohlfahrtsarrangements. Neue Wege in der Sozialwirtschaft. Baden-Baden. 